\title{
Position Effect Trial
}

National Cancer Institute

\section{Source}

National Cancer Institute. Position Effect Trial. NCI Thesaurus. Code C161477.

A type of trial designed to evaluate the effect of body position during and/or after administration of the investigational product. 